\title{
Shape Prediction of Large Deployable Antenna Structure on Orbit
}

\author{
By Kosei Ishimura ${ }^{1)}$, Tsuneo KII ${ }^{1)}$, Keiji Komatsu ${ }^{1)}$, Ken Goto ${ }^{1)}$, Ken $\mathrm{HIGUCHI}^{2)}$, \\ Kazuro MATSUMOTO ${ }^{3)}$, Shoichi IIKURA ${ }^{4)}$, Makoto YoshiHARA ${ }^{3)}$ and Masaharu TsuChiYA ${ }^{3)}$ \\ ${ }^{1)}$ The Institute of Space and Astronautical Science, JAXA, Sagamihara, Japan \\ ${ }^{2)}$ Department of Mechanical, Aerospace, and Materials Engineering, Muroran Institute of Technology, Muroran, Japan \\ ${ }^{3)}$ NEC TOSHIBA Space Systems, Ltd. (NTSpace), Fuchu, Japan \\ ${ }^{4)}$ NEC Aerospace Systems, Ltd., Fuchu, Japan
}

( Received June 27th, 2011)

\begin{abstract}
For the worst-case analysis of a space structure's shape on orbit, various factors such as effects of thermal deformation, aged deterioration, and material hysteresis should be considered. Furthermore, the parameters of each factor have some uncertainty, such as in material properties. Therefore, in shape prediction, the consideration of these various factors and their parameters' uncertainties leads to a combinatorial explosion. To solve this problem, the factors are classified by the mode shape of deformation. If the mode shapes of some factors have high correlation, those factors are categorized in the same group. Within each group, maximum and minimum deformations are analyzed considering the uncertainty in the parameters. Among the groups with low correlation, deformations are evaluated using a combination of the maximum and minimum deformations from each group. As a result, the combinations of factors and parameters are drastically reduced. Such a shape prediction method was applied to a large deployable antenna structure of ASTRO-G. In this study, the performance of this antenna is evaluated using GRASP analysis for the predicted antenna shapes.
\end{abstract}

Key Words: Deployable Structure, Antenna, Space Structure, Modular Structure

\section{Introduction}

Large antennas are used in various satellites for both communication and scientific observation, among other purposes $^{1-3)}$. Such space structures must be stowed in the launch vehicle. As a result, space structures larger than the fairing are required to be deployable. In addition, low areal density is required due to payload mass restriction. To realize a low areal density, a mesh-type antenna with a cable network was developed for the VLBI mission of MUSES-B ${ }^{1)}$ (Fig. 1).

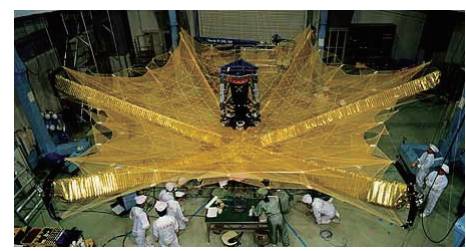

Fig. 1. MUSES-B antenna deployment test. (c) JAX

For large structures, gravitational effects are unavoidable in ground tests and can cause unpredicted deformations and internal loads, making various engineering tests difficult to perform. For example, an accurate representation of deployment behavior and structural shape cannot be obtained on the ground for large space structures over $10 \mathrm{~m}^{4}$. To solve this problem, the concept of a modularized structure was proposed $^{4}$, in which the whole structure consists of multiple smaller modules. The engineering tests can be carried out on a single module or a partial structure consisting of a few modules. The performance and characteristics of the whole structure are then predicted by analysis. Therefore, the verification and validation of the analytical model is very important for modularized structures. The large deployable reflector on ETS-VIII' ${ }^{2,5)}$ is an example of a modularized structure.

In the ASTRO-G project, an accurate antenna surface is required for scientific observation from $5 \mathrm{GHz}$ to $43 \mathrm{GHz}^{3}$. To improve surface accuracy, elastic deformation of radial ribs is used in the Large Deployable Antenna (LDA) of ASTRO-G ${ }^{3,6)}$ (Figs. 2 and 3) instead of a cable network. Figure 4 shows a schematic of a single module. In the conventional mesh-type antenna with a cable network ${ }^{1,5)}$, the parabolic curve of antenna surface is formed by segmented lines approximately. In the ASTRO-G LDA, the parabolic curve is formed by the deformed ribs. If the rib stiffness and cable tension are both designed appropriately, the deformed ribs will form a better approximation of the parabolic curve than segmented lines. The deployable truss in Fig. 4 is used as backup structure to support the antenna surface, which consists of mesh, cables, and ribs. The modules are connected at the edges of the backup truss rather than the antenna surface.

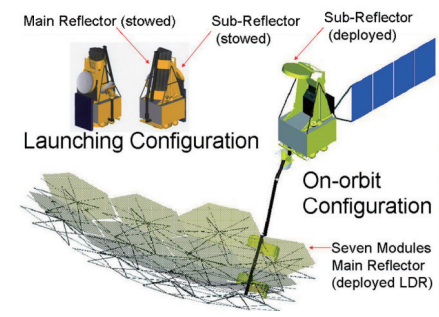

Fig. 2. ASTRO-G satellite configuration. 


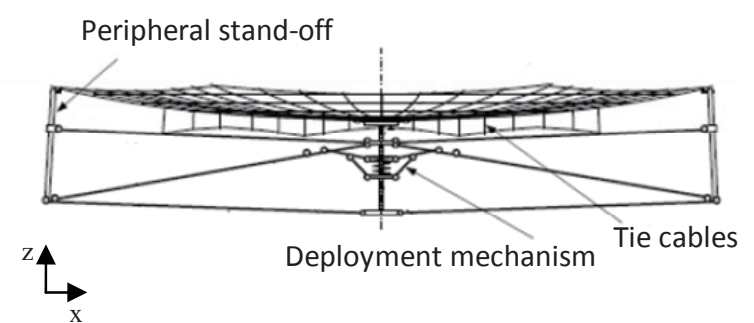

(a) side view

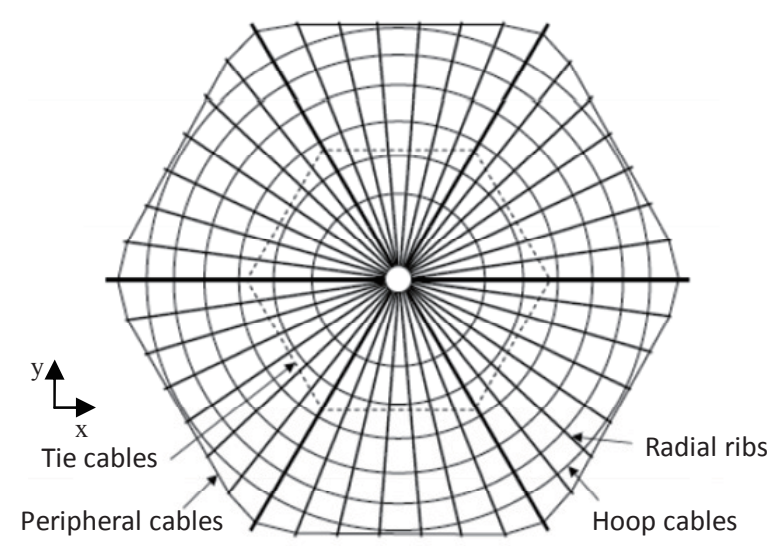

(b) top view

Fig. 3. Single ASTRO-G LDA module.

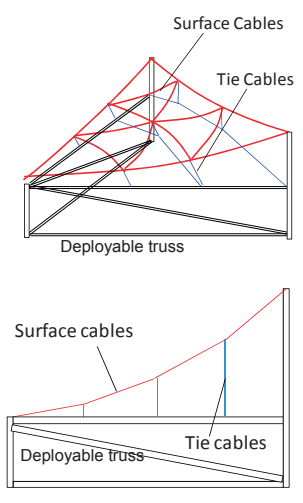

(a) cable network
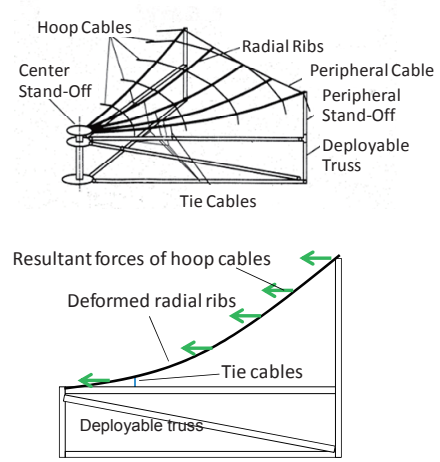

(b) deformed rib with cables
Fig. 4. Parabolic approximation of antenna surfaces (cross section of single module).

Although the antenna with elastically deformed ribs provides a good approximation of the parabolic surface, its shape is more dependent on stiffness compared to a conventional mesh-type antenna with a cable network. Therefore, if the stiffness of some structural member changes due to aged deterioration on orbit, the antenna shape also changes. To obtain an accurate prediction of the antenna's shape on orbit, a lot of material parameters, including time dependency $^{7}$, must be considered. In addition, the deformations are too large to apply linear theory. Therefore, to predict the shape, nonlinear analysis with geometric stiffness should be performed considering the various parameters' effects; this is an enormous computational undertaking.
In this paper, we propose a method for predicting the shapes of modularized LDAs that accounts for their nonlinearity but avoids combinatorial explosion. Worst-case analysis is performed for the ASTRO-G LDA using the proposed method. The worst-case analysis procedures are discussed in Sec. 2, and the analytical model verification is discussed in Sec. 3 . Finally, the worst-case analysis results are presented in Sec. 4.

\section{Shape Prediction Method for a Modularized LDA with Nonlinearity}

There are many surface errors that can affect modularized LDA performance, as shown in Table 1. These errors are divided into two classes based on spatial randomness: random error and systematic error. Usually, deployment repeatability is considered as a random error; however, deployment repeatability (single module) is mainly caused by cable hysteresis in the case of ASTRO-G, and the size and shape of the surface error is not very random, so it is treated as a systematic error. Furthermore, the systematic errors are divided into two subclasses based on uncertainty. Design error is constant and has no uncertainty. However, deployment repeatability (single module) and deformation on orbit have uncertainty in their parameters, such as in the aged deterioration of materials. The difference between initial error on orbit and deformation on orbit is the stability of the deformation. Initial error on orbit is an almost constant bias error. On the other hand, deformation on orbit changes depending on the attitude of satellite, the season, and degradation.

\begin{tabular}{|c|c|c|}
\hline \multirow{2}{*}{ Design error } & \multicolumn{2}{|c|}{ Linear approximation error of facet } \\
\hline & \multicolumn{2}{|c|}{ Pillow deformation error of facet } \\
\hline \multirow{2}{*}{$\begin{array}{l}\text { Assembly and } \\
\text { adjustment error }\end{array}$} & \multicolumn{2}{|c|}{ Adjustment error (single module) } \\
\hline & \multicolumn{2}{|c|}{ Assembly error } \\
\hline \multirow{4}{*}{ Initial error on orbit } & \multicolumn{2}{|c|}{ Zero-G cancellation error } \\
\hline & \multicolumn{2}{|c|}{ Deployment repeatability (single module) } \\
\hline & \multicolumn{2}{|c|}{ Deployment repeatability (assembly error) } \\
\hline & \multicolumn{2}{|c|}{$\begin{array}{l}\text { Deformation in vacuum due to moisture } \\
\text { expansion on ground }\end{array}$} \\
\hline \multirow{4}{*}{ Deformation on orbit } & Therm & rmation \\
\hline & Aged deteri & of materials \\
\hline & \multicolumn{2}{|c|}{ Creep } \\
\hline & Systematic error & Random error \\
\hline
\end{tabular}

\subsection{Random errors}

Although the shapes of random surface errors cannot be predicted, the standard deviations of Gaussian surface errors can be predicted and refined based on ground test results. Adjustment error (single module) and deformation in vacuum (single module) can be measured in ground tests. However, assembly error for the whole structure cannot be accurately measured on the ground due to gravitational effects. To obtain the assembly error and deployment repeatability for the whole structure, analysis must be used. The details of the analysis are discussed in Sec. 3. 


\subsection{Systematic errors}

For systematic errors, constant errors without uncertainty can be dealt with easily. Because deployment repeatability (one module) and deformation on orbit have uncertainty in their parameters, multiple cases should be analyzed to obtain the worst case. Factors of these systematic errors with uncertainty are listed in Table 2 . To predict the worst case, a combination of these 14 factors, avoiding duplication of thermal deformation (BOL/EOL), should be considered; however, this leads to combinatorial explosion $\left(2^{14} \fallingdotseq 1.6 \times\right.$ $\left.10^{4}\right)$.

To reduce the number of combinations, we classify the factors by the mode shape of deformation. If the mode shapes of some factors have high correlation, those factors are categorized in the same group. Representative deformations of each mode (whole antenna surface and central radial rib) along the $\mathrm{z}$ axis (normal to the antenna surface) are shown in Fig. 5. In the ASTRO-G case, the factors are divided into three groups: CF cable mode, radial rib mode, and tie cable mode. In the same group, maximum and minimum deformations are analyzed considering the uncertainty in the parameters and assuming that the parameters' effects on the surface error are monotone, as shown in Fig. 6. Based on this assumption, the max or min deformation corresponds to the max or min parameter.

Among the groups with low correlation, the deformation is evaluated using a combination of the maximum and minimum overall deformations from each group. However, the effect of deployment repeatability is considered using three coefficients: $0.5,1.0$, and 1.5. These coefficients are applied to the largest deformation obtained in deployment repeatability tests. Because the deformation due to the error is large and is a major contributor to the overall surface error, the simple assumption of monotony includes some risk. Therefore, the three coefficients are used when considering deployment repeatability. As a result, the combinations of factors and parameters are drastically reduced $\left(3 \times 2^{3}=24\right)$.

For these 24 cases, the surface deformation is analyzed including design errors. Based on the obtained surface deformation, the antenna gain loss is evaluated by GRASP (General Reflector and Antenna Analysis Software Package). The equivalent RMS $\sigma_{s y s}$ is then calculated from the antenna gain loss by applying Ruze's equation. Finally, the systematic errors (the equivalent RMS $\sigma_{s y s}$ ) are integrated with the random errors.
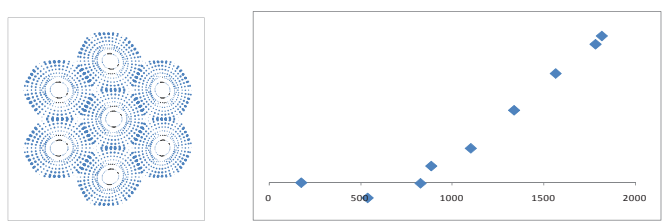

(a) CF cable mode
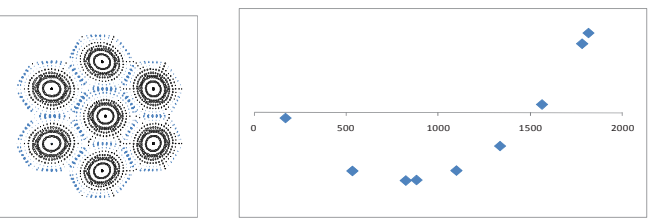

(b) radial rib mode
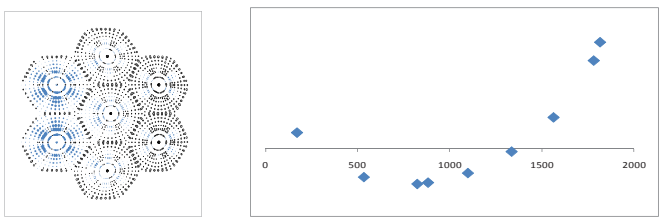

(c) tie cable mode

Fig. 5. Representative deformation of each mode along the $\mathrm{z}$ axis. (The blue circle means + and the white circle means -. Circle size is proportional to displacement. The left figures show the deformation of the whole antenna surface. The right figures show the deformation of the center radial rib. The horizontal axis in the right figure indicates the radial position.)

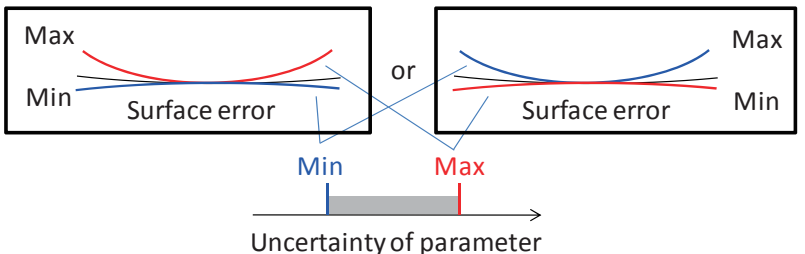

Fig. 6. Monotony of the parameters' effects on surface error for a single module.

Table 2. Factors of the systematic errors with uncertainty and their correlations

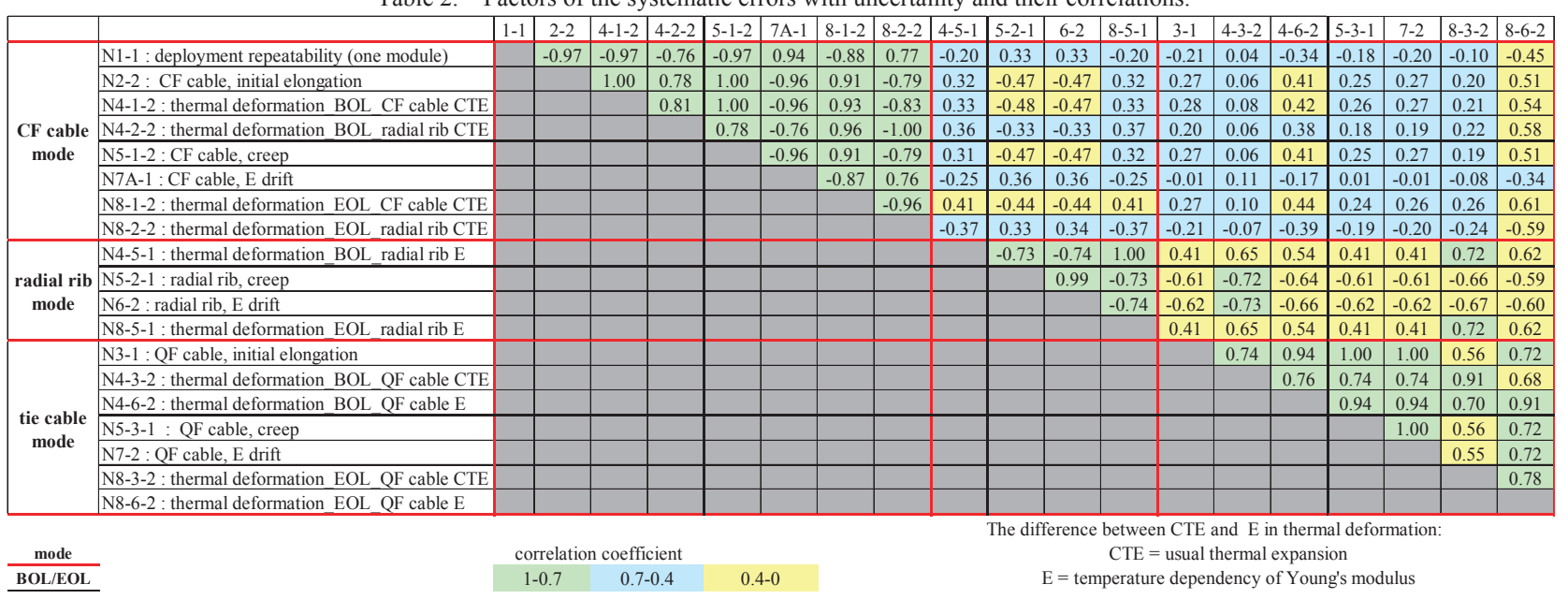




\subsection{Evaluation-integrated surface accuracy}

The reflectance of a reflector is estimated by Ruze's equation:

$$
R=\exp \left[-\left(\frac{4 \pi}{\lambda}\right)^{2} \sum_{k} \sigma_{k}^{2}\right]
$$

where $\lambda$ is the wavelength and $\sigma_{k}$ is the standard deviation of the surface error caused by the $\mathrm{k}^{\text {th }}$ independent Gaussian factor. Applying Ruze's equation, the integrated surface accuracy, $\sigma_{i t g}$, is estimated by the following equation:

$$
\sigma_{i t g}^{2}=\sigma_{s y s}^{2}+\sum_{k} \sigma_{k_{-} \text {rand }}^{2}
$$

where $\sigma_{k \text { rand }}$ is the standard deviation of the surface error caused by the $\mathrm{k}^{\text {th }}$ random factor in Sec. 2.1.

\section{Verification of Analytical Model}

Because the performance and characteristics of the whole structure are predicted by analysis for a modularized structure, the verification of the analytical model is very important. The analytical model is verified by ground testing, and the verification is carried out in three steps (Fig. 7).

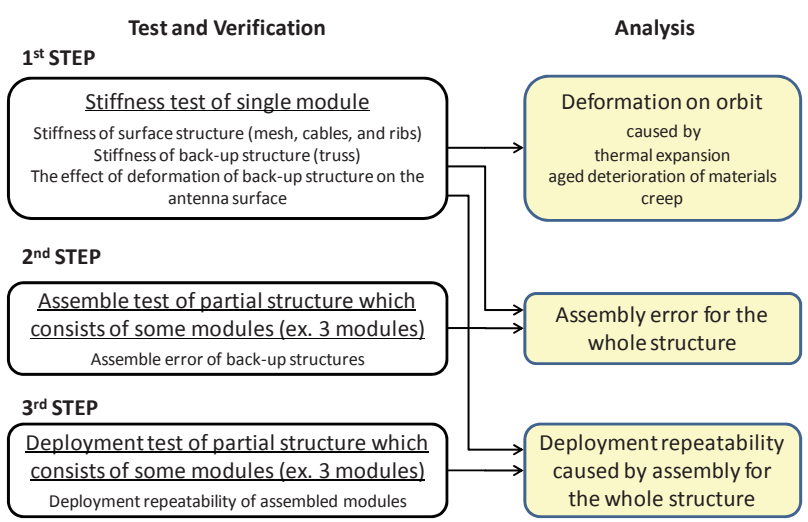

Fig. 7. Test and verification process.

In the first step, the stiffnesses of the surface structure (mesh, cables, and ribs) and backup structure (truss) are evaluated through stiffness testing (single module) and are then correlated with the analytical model of single module. This step is a standard process in obtaining an accurate analytical model. The effect of the backup structure deformation on the antenna surface is also evaluated in this test. The relationship between the backup structure and the antenna surface is very important because the modules are connected by the truss edges and deformations of adjoining modules are transferred to the antenna surface through the truss. Examples of the stiffness test results and corresponding analysis results are shown in Fig. 8.

In the next step, the assembly test of the partial structure, which consists of multiple modules, is performed to evaluate the assembly error of the backup structure. The actual connecting point locations may not coincide with the intended locations due to manufacturing error, residual adjustment error, and so on. When such modules with geometrical discrepancies are assembled, the backup structures deform and cause the antenna surface to also deform. This is called assembly error (Table 1). The partial structure assembly test uses the truss structures of three modules, which is the smallest assembled configuration with a closed loop (Fig. 9); furthermore, undesired stresses generated by gravity are small in this case because the three truss modules can be suspended by three wires, which also define a plane (Fig. 10). To compensate for gravity, the difference between the two types of deformation is used: nominal residual error in the adjustment and large residual error. An example of the assembly test results for the partial structure consisting of three ETS-VIII modules is shown in Fig. 11 along with the corresponding analytical results. In the analysis, residual adjustment error (position error of the truss connecting points) is modeled by the thermal deformation of the connecting points. Through this step, the analytical model of the assembled truss structure is verified. By combining this model with the verified single module analytical model from the first step, the surface error of the whole structure caused by the assembly process can be analyzed.

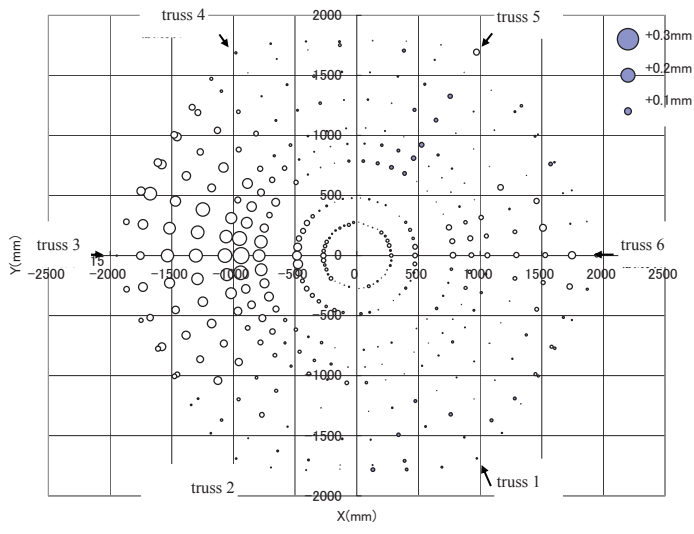

(a) stiffness test results

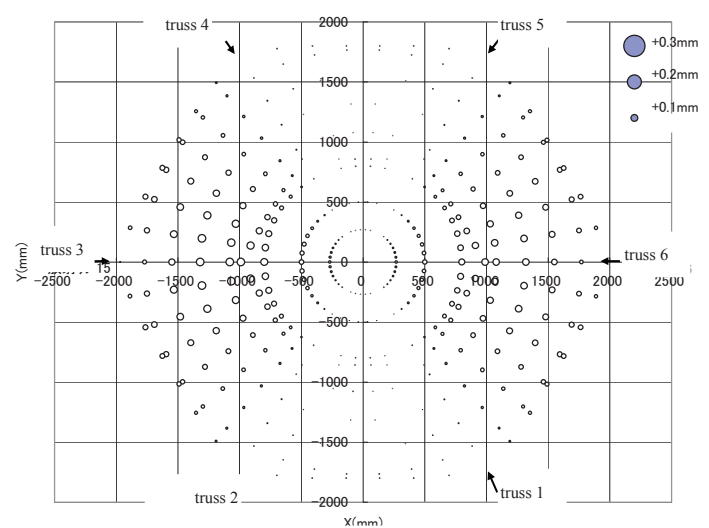

(b) analysis results

Fig. 8. Surface displacement along the $\mathrm{z}$ axis (normal to the surface) against the radial load applied at the edges of trusses 3 and 6 (blue circle means + , white circle means -, circle size is proportional to the displacement). 


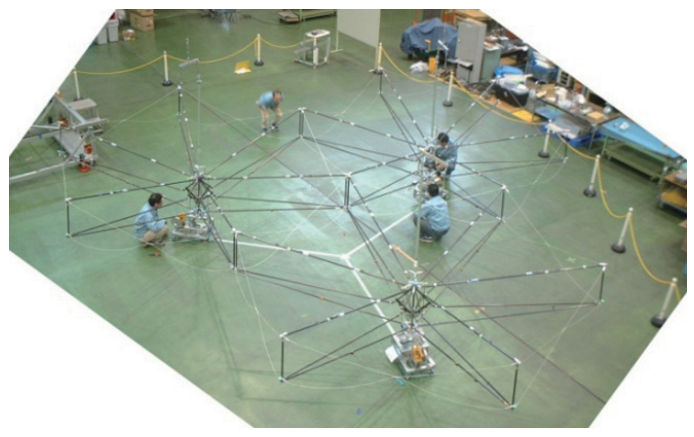

Fig. 9. Assembly test of three truss modules.

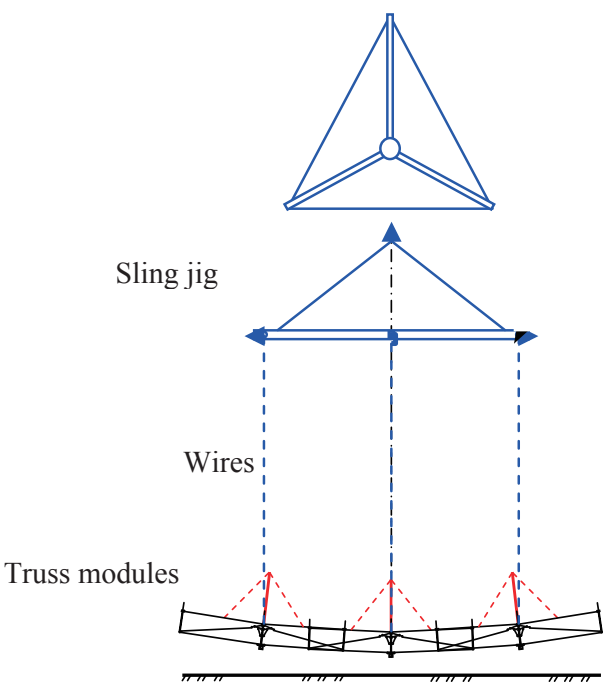

Fig. 10. Assembly test article suspended by wires (red lines show tension load mechanisms, which are used instead of hoop cables and mesh to transfer loads to the truss).

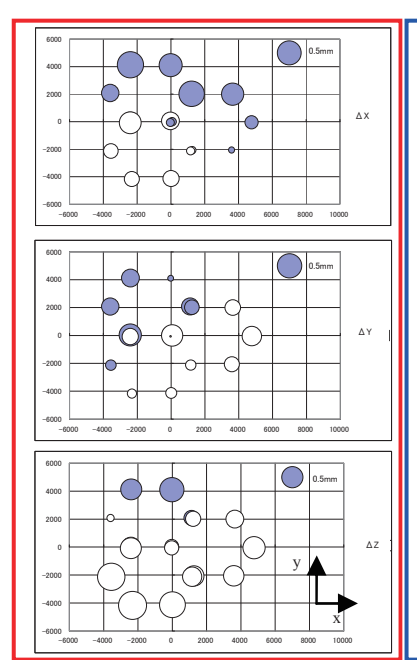

(a) Assembly test results

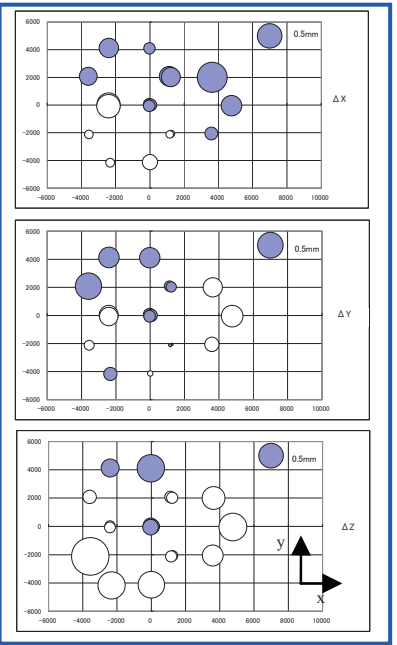

(b) Analysis results
Fig. 11. Truss displacements along $\mathrm{x}, \mathrm{y}$, and $\mathrm{z}$ axes due to assembly (blue circle means + , white circle means -, circle size is proportional to displacement).

In the last step, deployment repeatability (assembly error) (Table 1) is verified by the deployment test of the partial structure consisting of multiple truss modules. This is different from deployment repeatability (single module) because deployment repeatability (assembly error) is a random error due to gaps at the connecting points in the assembly. These gaps are also modeled by the thermal deformation of the connecting points in the analysis. Figure 12 shows the results of the deployment test and analysis using the Monte Carlo method, in which the gaps at the connecting points are assigned a random uniform value within the tolerance (108 cases). In the deployment test, the assembled trusses were deployed three times, and the truss deformation was measured four times, including a measurement of the initial state. The truss deformation (mm RMS) was evaluated against the nominal analysis case in the analysis. In the test results, it is evaluated against the first test result.

Assembly error and deployment repeatability (assembly error) are estimated using the ASTRO-G analytical model. The results are summarized in the next section.

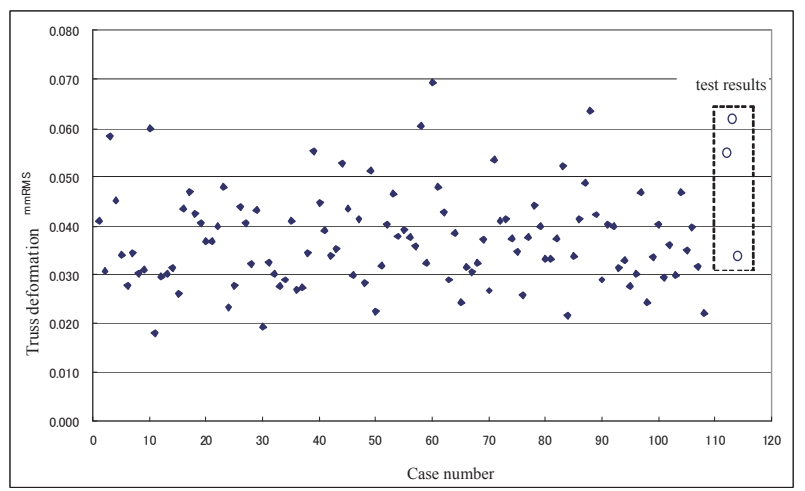

Fig. 12. Deployment repeatability caused by assembly.

\section{Shape Prediction of Modularized LDA}

Shape predictions for the modularized ASTRO-G LDA were performed using the verified analytical model from Sec. 3. The predicted error is summarized in Table 3. The systematic surface errors were predicted using the method mentioned in Sec. 2.3. The random surface errors, which cannot be measured (assembly error and deployment error caused by assembly), were predicted by the Monte Carlo simulation shown in Sec. 3. The integrated surface accuracy $\sigma_{i t g}$ was estimated by Eq. (2). As a result, the surface error of the ASTRO-G LDA was estimated at $0.57 \mathrm{~mm}$ RMS at BOL and $0.75 \mathrm{~mm}$ RMS at $1 / 2 \mathrm{EOL}$ in the nominal case; this is sufficient surface accuracy for scientific observation. Although the surface error of the LDA was estimated at 0.67 $\mathrm{mm}$ RMS at BOL and $1.16 \mathrm{~mm}$ RMS at $1 / 2 \mathrm{EOL}$ in the worst case, the probability of the worst case is extremely low: it will only occur if all parameters with uncertainty have the worst value. 
Table 3. Shape prediction of modularized LDA.

\begin{tabular}{|c|c|c|c|}
\hline \multicolumn{3}{|c|}{ Error Source } & $\begin{array}{l}\text { Prediction } \\
\text { (equivalent }\end{array}$ \\
\hline \multirow{2}{*}{ Design error } & \multicolumn{2}{|c|}{ linear approximation error of facet } & 0.12 \\
\hline & \multicolumn{2}{|c|}{ pillow deformation error of facet } & 0.20 \\
\hline \multirow{3}{*}{$\begin{array}{l}\text { Assembly and } \\
\text { adjustment error }\end{array}$} & one module & adjustment error & 0.20 \\
\hline & \multirow{2}{*}{ assembly error } & assembly error & 0.29 \\
\hline & & measurement error & 0.10 \\
\hline \multirow{4}{*}{$\begin{array}{l}\text { Initial error } \\
\text { on orbit }\end{array}$} & \multicolumn{2}{|c|}{ zero-G cancellation error } & 0.05 \\
\hline & \multirow{2}{*}{$\begin{array}{l}\text { deployment } \\
\text { repeatability }\end{array}$} & one module* & 0.59 \\
\hline & & assembly error & 0.07 \\
\hline & \multicolumn{2}{|c|}{ deformation in vaccum } & 0.19 \\
\hline \multirow{2}{*}{$\begin{array}{c}\text { Deformation } \\
\text { on orbit }\end{array}$} & \multicolumn{2}{|c|}{ BOL* } & 0.22 \\
\hline & \multicolumn{2}{|c|}{$1 / 2 \mathrm{EOL}(1.5 \mathrm{yr}) *$} & 0.70 \\
\hline \multicolumn{4}{|c|}{ Total Error } \\
\hline \multirow{2}{*}{ GRASP analysis } & \multicolumn{2}{|c|}{ BOL } & $\begin{array}{c}\mathbf{0 . 5 7} \text { (nominal) } \\
0.67 \text { (worst) }\end{array}$ \\
\hline & \multicolumn{2}{|c|}{ 1/2 EOL (1.5 yr) } & $\begin{array}{c}\mathbf{0 . 7 5} \text { (nominal) } \\
1.16 \text { (worst) }\end{array}$ \\
\hline
\end{tabular}

\section{Conclusion}

A novel method for predicting the shape of modularized LDAs with nonlinearity was proposed; this method avoids combinatorial explosion. Furthermore, verification procedures for the analytical model of the modularized structure were also presented. The prediction method and verification procedures were also applied to the ASTRO-G LDA. As a result, it was shown that the LDA can sufficiently maintain its shape on orbit for the purpose of scientific observation.

\section{Acknowledgments}

The authors sincerely appreciate the cooperation of NEC, NTSpace, NEC Aerospace Systems, and ASTRO-G project members.

\section{References}

1) Natori, M.C., Takano, T., Inoue, T. and Noda, T.: Design and Development of a Deployable Mesh Antenna for MUSES-B Spacecraft, Proceedings of Structures, Structural Dynamics, and Materials Conference, AIAA-1993-1460.

2) Uchimaru, K., Nakamura, K., Tsujihata, A., Miyasaka, A. and Meguro, A.: Large Deployable Reflector on ETS-VIII (Second Report), Proceedings of AIAA 18th International Communications Satellite Systems Conference and Exhibit, AIAA-2000-1144.

3) Hirabayashi, H., Murata, Y., Edwars, P.G., Asaki, Y., Mochizuki, N., Inoue, M., Umemoto, T., Kameno, S. and Kono, Y.: On the Near-term Space VLBI Mission VSOP-2, Proceedings of the $7^{\text {th }}$ European VLBI Network Symposium, 2004.

4) Mitsugi, J. and Yasaka, T.: Deployable Modular Mesh Antenna Concept and Feasibility, Proceedings of $17^{\text {th }}$ International Symposium on Space Technology and Science, 1990, pp. 599-604.

5) Meguro, A., Shintate, K., Usui, M. and Tsujihata, A.: In-orbit Deployment Characteristics of Large Deployable Antenna Reflector Onboard Engineering Test Satellite VIII, Acta Astronautica, 65 2009, pp. 1306-1316.

6) Higuchi, K., Kishimoto, N., Kawahara, K., Mizuno, T., Tachikawa, S., Murata, Y., Tsuboi, M., Saito, H., Ogawa, H., Kimura, S.,
Ujihara, H., Tanaka, H., Meguro, A., Yoshihara, M. and Iikura, S.: Large Deployable Antenna Structure for VSOP-2 Mission, $59^{\text {th }}$ International Aeronautical Congress, IAC-08-C2.2.5

7) Goto, K., Kii, T., Yoshihara, K., Ishimura, K., Komatsu, K., Higuchi, K., Maruyama, T., Ikeda, Y., Matsumoto, K., Yoshihara, M. and Tsuchiya, M.: Long Term Durability of a Rib and Cable Tensioned Structure for High Accuracy Large Deployable Antenna Reflector, $28^{\text {th }}$ International Symposium on Space Technology and Science, 2011. 